\title{
miR-124a restoration inhibits glioma cell proliferation and invasion by suppressing IQGAP1 and $\beta$-catenin
}

\author{
SHAO-HUA LU, XING-JUN JIANG, GE-LEI XIAO, \\ DING-YANG LIU and XIAN-RUI YUAN
}

\begin{abstract}
The Institute of Skull Base Surgery and Neurooncology at Hunan, Xiangya Hospital, Central South University, Changsha, Hunan 410008, P.R. China
\end{abstract}

Received May 23, 2014; Accepted July 24, 2014

DOI: $10.3892 /$ or.2014.3455

\begin{abstract}
A number of microRNAs have been identified to be important regulators of tumorigenesis. Previous research has shown that miR-124 is abundantly expressed in normal brain tissue; however, only a few reports have focused on the biological impact of miR-124 on glioma cells, and the underlying mechanisms need to be elucidated. Therefore, we investigated the effect of miR-124a on glioma cell proliferation and invasion; furthermore, the underlying molecular mechanism was examined. The present study demonstrated that miR-124a expression was downregulated in human glioma tissues, and its expression level was negatively correlated with the pathological grade of the glioma. Restoration of miR-124a inhibited glioma cell proliferation and invasion in vitro. Furthermore, we found that miR-124a directly targeted and suppressed IQ motif containing GTPase activating protein 1 (IQGAP1), a well-known regulator of actin dynamics and cell motility. RNA interference assay showed that IQGAP1 knockdown led to downregulation of $\beta$-catenin and downstream cyclin D1. Taken together, our study revealed that miR-124a could inhibit glioma cell proliferation and invasion by blocking the expression of the IQGAP1 gene and downstream $\beta$-catenin and cyclin D1. This research may provide a useful molecular therapy for gliomas.
\end{abstract}

\section{Introduction}

Gliomas account for $80 \%$ of all primary brain and central nervous system malignancies (1). Gliomas are difficult to be cured by surgical resection or radiotherapy, and the median survival of patients with gliomas is only 12-15 months (2). Therefore, it is necessary to elucidate the underlying mecha-

Correspondence to: Dr Xian-Rui Yuan, The Institute of Skull Base Surgery and Neurooncology at Hunan, Xiangya Hospital, Central South University, 87 Xiangya Road, Changsha, Hunan 410008, P.R. China

E-mail: dryxr111@163.com

Key words: miR-124a, proliferation, invasion, IQGAP1, $\beta$-catenin nisms involved in glioma development and to discover new targets for the treatment of gliomas.

Accumulating evidence suggests that changes in the expression of microRNAs (miRNAs) are associated with cancer development (3), and a number of miRNAs have been identified to be important regulators of tumorigenesis $(4,5)$.

Previous research has shown that miR-124 is abundantly expressed in normal brain tissue (6); however, only a few reports have focused on the biological impact of miR-124 on glioma cells (7) and the underlying mechanisms need to be elucidated.

In the present study, we investigated the role of miR-124a in glioma proliferation and invasion. The in vivo study demonstrated that expression of miR-124a was downregulated in glioma tissues and in highly malignant glioma cells. Restoration of miR-124a or the knockdown of IQGAP1 in vitro inhibited glioma cell proliferation and invasion. Furthermore, we confirmed that miR-124a could inhibit glioma cell proliferation and invasion by blocking the expression of the IQGAP1 gene and downstream $\beta$-catenin and cyclin D1.

\section{Materials and methods}

Cell culture. Human glioma cell lines (U251, U343, U87, SF126 and SF76) were purchased from the American Type Culture Collection (ATCC; Manassas, VA, USA) and cultured in Dulbecco's modified Eagle's medium (DMEM; Gibco, Grand Island, NY, USA) supplemented with $10 \%$ fetal bovine serum (Gibco) at $37^{\circ} \mathrm{C}$ in a humidified atmosphere of $5 \% \mathrm{CO}_{2}$.

Tissue samples. This study was approved by the Ethics Committee of the Third Xiangya Hospital of Central South University. All patients provided written informed consent in compliance with the code of ethics of the World Medical Association (Declaration of Helsinki). Human glioma samples and adjacent normal tissue samples were collected from 20 glioma patients who underwent surgery at the Neurosurgery Department of the Third Xiangya Hospital of Central South University (Changsha, Hunan, China). At the time of diagnosis, 10 patients had early stage (I, II) whereas the other 10 patients had stage III and IV glioma. The tissue samples were obtained from surgery and immediately frozen in liquid nitrogen. 
Real-time PCR. Total RNAs were isolated from cells and tissues using TRIzol (Invitrogen, Carlsbad, CA, USA) according to the manufacturer's instructions. Reverse transcription was performed using a RevertAid ${ }^{\mathrm{TM}}$ First Strand cDNA Synthesis kit (Fermentas, Vilnius, Lithuania). Real-time PCR reaction was carried out in a 7900 Sequence Detection System (Applied Biosystems, Foster City, CA, USA) using a SYBR-Green PCR kit (Applied Biosystems). The relative mRNA expression level was calculated by the comparative $\mathrm{Ct}$ method.

Western blotting. Tissues and cells were lysed by ice-cold protein extraction buffer (150 mM Tris-HCl, pH 7.4, $120 \mathrm{mM}$ $\mathrm{NaCl}, 2 \mathrm{mM}$ EDTA, $50 \mathrm{mM}$ sodium fluoride, $0.2 \% \mathrm{SDS}, 1 \%$ Nonidet P-40, $100 \mathrm{mM}$ sodium vanadate and $1 \mathrm{mM}$ phenylmethylsulfonyl fluoride). Proteins were quantitated using the BCA kit (Beyotime, Shanghai, China). The protein samples were separated in $10 \%$ SDS-PAGE and transferred onto a nitrocellulose membrane (Millipore, Billerica, MA, USA). The membranes were blocked overnight in 5\% non-fat milk and then incubated with the primary antibody [IQGAP1 and $\beta$-catenin antibodies (Abcam, Cambridge, MA, USA); p- $\beta$-catenin and cyclin D1 antibodies (Cell Signaling Technology, Inc., Beverly, MA, USA); GAPDH antibody (Santa Cruz Biotechnology, Inc., Santa Cruz, CA, USA)] for $1 \mathrm{~h}$ at room temperature. After washing with PBS, the membranes were incubated with the secondary antibody [HRP-conjugated goat anti-mouse IgG and HRP-conjugated goat anti-rabbit IgG (Santa Cruz Biotechnology, Inc.)] for $1 \mathrm{~h}$ at room temperature. Detection was performed using a chemiluminescence-based detection system (ECL western blotting kit; Pierce Biotechnology, Inc., Rockford, IL, USA).

Immunofluorescence. Cells were washed with PBS and fixed in $4 \%$ paraformaldehyde solution for $1 \mathrm{~h}$. After permeabilization with $0.2 \%$ Triton $\mathrm{X}-100$ at $4^{\circ} \mathrm{C}$ for $1 \mathrm{~h}$, cells were blocked with goat serum and then incubated with the primary antibody ( $\beta$-catenin antibody) for $1 \mathrm{~h}$ at $37^{\circ} \mathrm{C}$. Subsequently, the secondary antibody [Alexa Fluor 488-labeled anti-rabbit IgG (Cell Signaling Technology Inc.)] was added, and the cells were incubated at $37^{\circ} \mathrm{C}$ for $1 \mathrm{~h}$. DAPI (Santa Cruz Biotechnology, Inc.) was used to label the nucleus, and the cells were visualized by fluorescence microscopy (Nikon, Tokyo, Japan).

Transfection. The miR-124a mimic and siRNAs were synthesized by Biomics Biotechnologies Inc. (Nantong, Jiangsu, China). Cells were transfected with the miR-124a mimic or siRNAs using Lipofectamine 2000 (Invitrogen, Carlsbad, CA, USA) following the manufacturer's instructions. After $6 \mathrm{~h}$, the cultures were replaced with fresh medium.

Luciferase assay. The pRL-TK Renilla luciferase reporter vector, pmirGLO luciferase reporter vector and Dual-luciferase reporter assay system were purchased from Promega (Madison, WI,USA). Reporter plasmids containing the 3'UTR of IQGAP1 (pmirGLO-IQGAP1) were co-transfected with the NC mimic or the miR-124a mimic into cells. pRL-TK Renilla luciferase reporter vector was used as an internal control. Luciferase activity was measured by the Dual-luciferase reporter assay system. Results were expressed as the firefly luciferase activity normalized to Renilla luciferase activity.
MTT assay. The MTT assay was used to assess cell proliferation. Cells were seeded in 96-well plates and allowed to grow for $24 \mathrm{~h}$. After transfection, $50 \mu \mathrm{l}$ MTT solution was added and incubated at $37^{\circ} \mathrm{C}$ for $4 \mathrm{~h}$. After dissolving the formazine granulars with $150 \mu \mathrm{l}$ DMSO, the optical density (OD) at $570 \mathrm{~nm}$ was measured using a microplate reader (Ascent 354; Thermo Labsystems, Waltham, MA, USA).

Transwell-Matrigel invasion assay. Transwell inserts (Corning) were coated with Matrigel (BD Biosciences, Franklin Lakes, NJ, USA) at $37^{\circ} \mathrm{C}$ for $30 \mathrm{~min}$. The cells were suspended in serum-free medium at a final density of $5 \times 10^{4}$ cells $/ \mathrm{ml}$ and seeded to the upper chambers. Cell medium containing $10 \%$ FBS was added to the lower chambers. After incubation at $37^{\circ} \mathrm{C}$ for $12 \mathrm{~h}$, non-invaded cells were removed by a cotton swab. The invaded cells were fixed in $95 \%$ ethanol for $20 \mathrm{~min}$, followed by staining with hematoxylin for $10 \mathrm{~min}$. The number of invaded cells was counted under an inverted microscope (Nikon).

Statistical analysis. The Student's t-test was used to analyze differences between two groups. Data are expressed as the mean \pm SD of at least three independent experiments. $\mathrm{P}<0.05$ was defined as statistically significant.

\section{Results}

Expression of miR-124a in glioma tissue samples and glioma cell lines. The expression level of miR-124a in the clinical tissue specimens was determined by real-time PCR. The clinical glioma tissue samples were divided into two groups: low grade gliomas (grades I-II) and high grade gliomas (grades III-IV). As shown in Fig. 1A, the expression level of miR-124a in the glioma tissues was decreased when compared with the level in the normal tissues, and the expression level of miR-124a in the high grade gliomas was lower than that in the low grade gliomas.

We detected the expression level of miR-124a in a series of human glioma cell lines (U251, U343, U87, SF126, SF767). The results revealed that the expression level of miR-124a was lowest in the highly malignant glioma cell line U87 (Fig. 1B).

Expression of IQGAPI, $\beta$-catenin and phospho- $\beta$-catenin in glioma tissue samples and glioma cell lines. Expression of IQGAP1, $\beta$-catenin and phospho- $\beta$-catenin in the glioma tissue samples was determined by western blot analysis. The results revealed that the relative protein levels of IQGAP1 and $\beta$-catenin were increased in the glioma tissues when compared with the levels in the normal tissues. However, the relative protein level of phospho- $\beta$-catenin in the glioma tissues was lower than that in the normal tissues (Fig. 1C).

In addition, expression levels of IQGAP1, $\beta$-catenin and phospho- $\beta$-catenin were examined in a series of human glioma cell lines. As shown in Fig. 1D, U87 cells had high levels of IQGAP1 and $\beta$-catenin, but a low expression level of phospho- $\beta$-catenin. Based on the low expression level of miR-124a and high expression level of IQGAP1, we conducted miR-124a restoration and IQGAP1 knockdown on U87 cells for the following study in vitro. 

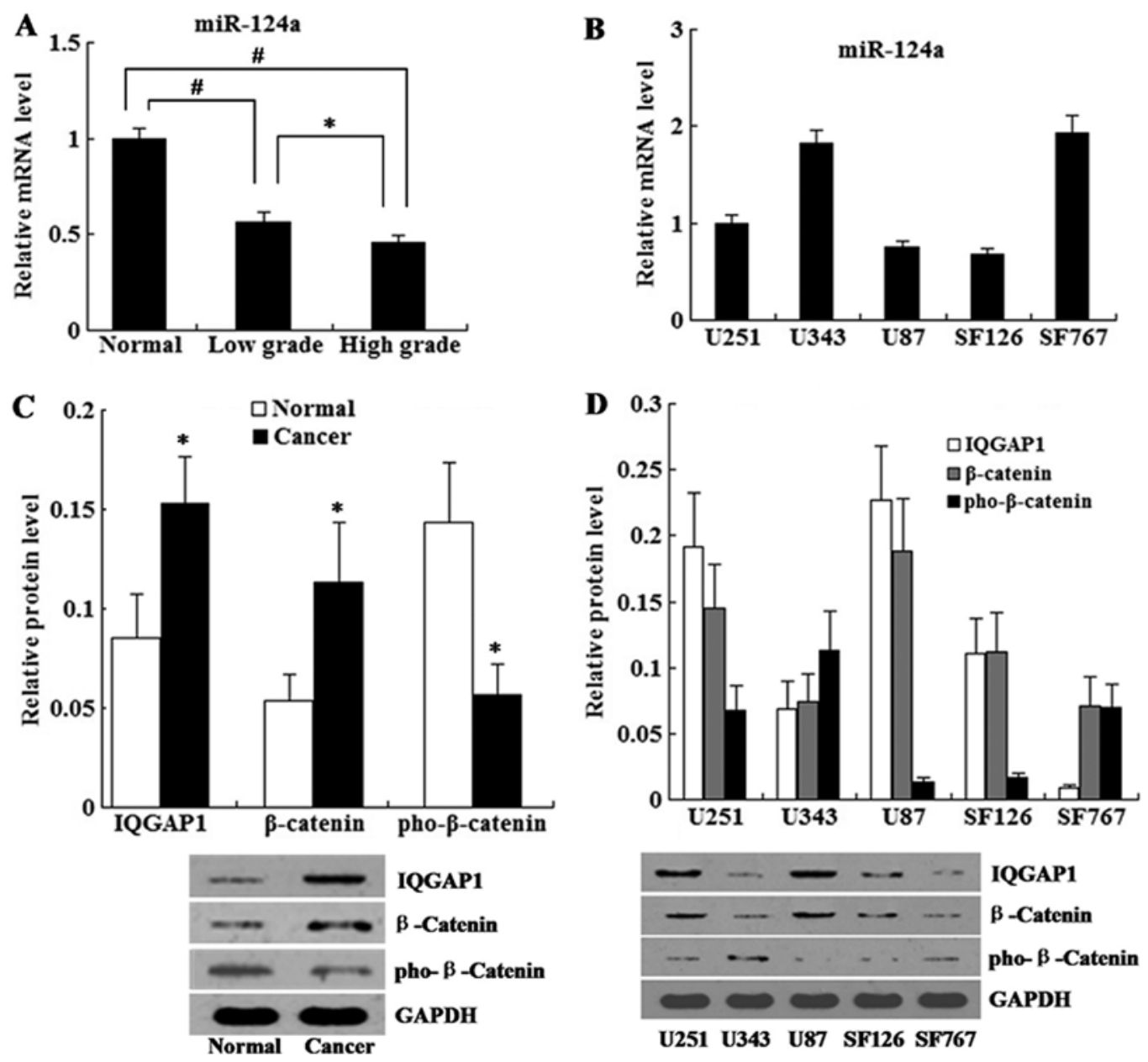

Figure 1. Expression of miR-124a, IQGAP1, $\beta$-catenin and phospho- $\beta$-catenin in glioma tissue samples as well as in glioma cell lines. (A) Real-time PCR analysis of the relative expression level of miR-124a in glioma tissue samples. (B) Real-time PCR analysis of the relative expression levels of miR-124a in the glioma cell lines. (C) Western blot analysis of relative expression levels of IQGAP1, $\beta$-catenin and phospho- $\beta$-catenin in glioma tissue samples, normalized to GAPDH. (D) Western blot analysis of relative expression levels of IQGAP1, $\beta$-catenin and phospho- $\beta$-catenin in the glioma cell lines, normalized to GAPDH. ${ }^{*} \mathrm{P}<0.05$ and ${ }^{\#} \mathrm{P}<0.01$ vs. the normal group. miR-124a, microRNA-124a; IQGAP1, IQ motif containing GTPase activating protein 1.
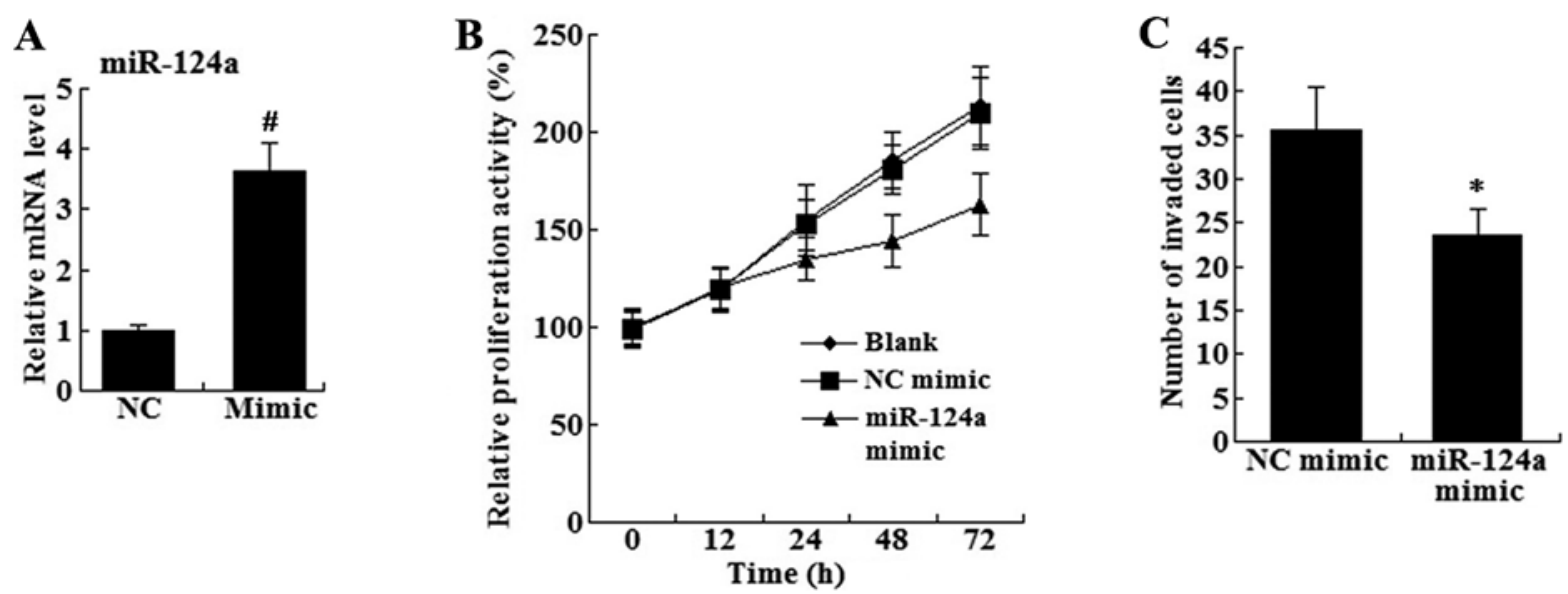

Figure 2. Effect of miR-124a restoration on cell proliferation and invasion. (A) Expression of miR-124a in U87 cells transfected with the NC mimic or the miR-124a mimic. (B) Proliferation rate of U87 cells transfected with the NC mimic or the miR-124a mimic as determined by MTT assay. (C) The numbers of invaded U87 cells transfected with the NC mimic or the miR-124a mimic. " $\mathrm{P}<0.05$ and ${ }^{~} \mathrm{P}<0.01$ vs. the control group. miR-124a, microRNA-124a.

miR-124a restoration inhibits cell proliferation and invasion. To investigate the effects of miR-124a restoration on glioma cells, the miR-124a mimic was transfected into U87 cells. As shown in Fig. 2A, the expression level of miR-124a was higher in the miR-124a mimic group than that in the control group. 

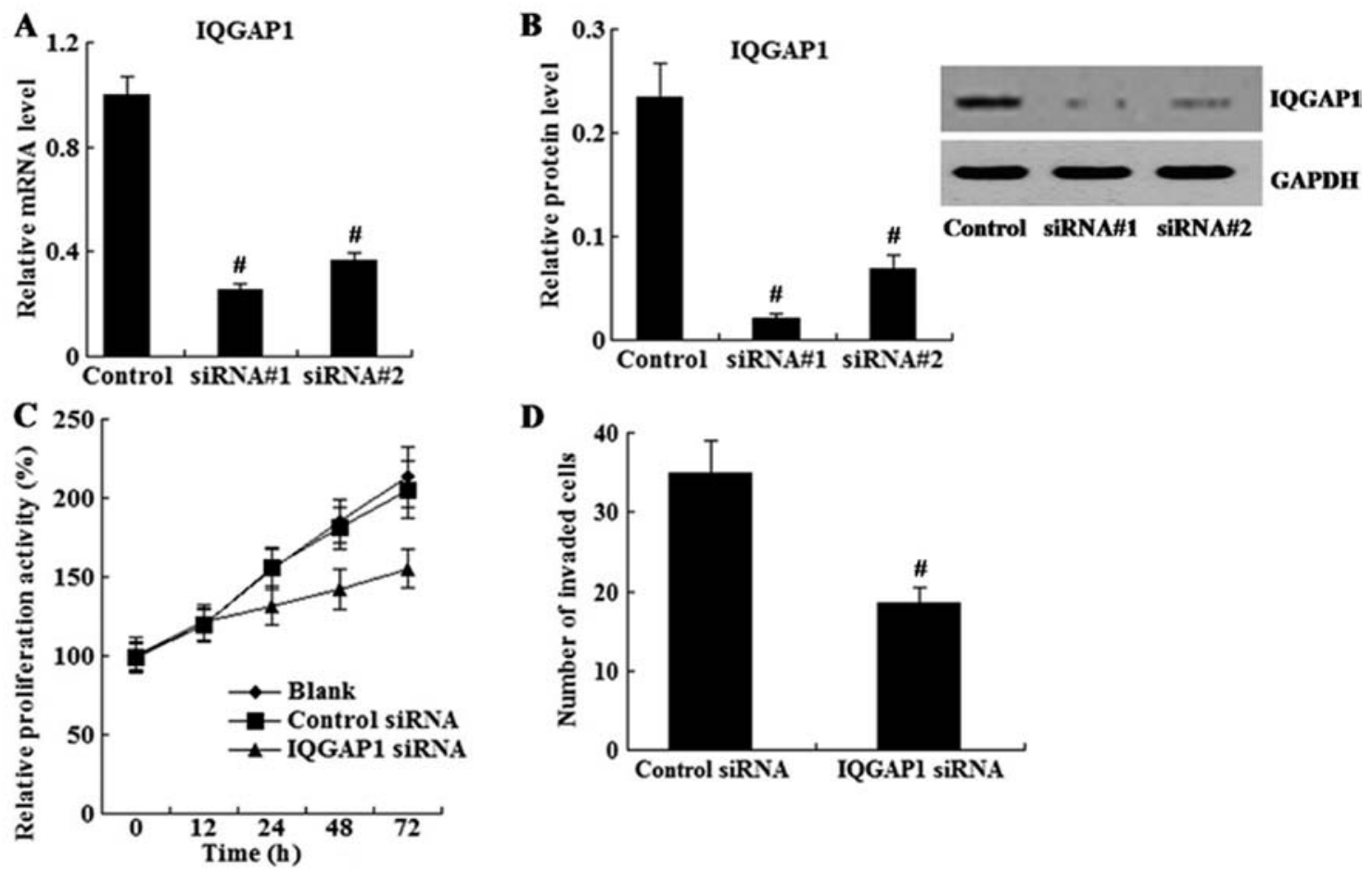

D

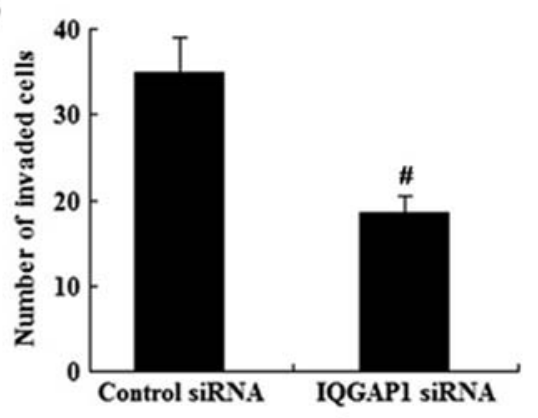

Figure 3. Effect of IQGAP1 knockdown on cell proliferation and invasion. (A) Relative mRNA level of IQGAP1 in U87 cells transfected with IQGAP1 siRNAs. (B) Western blot analysis of the relative protein level of IQGAP1 in U87 cells transfected with IQGAP1 siRNAs. (C) Proliferation rate of U87 cells transfected with the control siRNA or the IQGAP1 siRNA\#1 as determined by MTT assay. (D) The numbers of invaded U87 cells transfected with the control siRNA or the IQGAP1 siRNA\#1. "P $<0.01$ vs. the control group. IQGAP1, IQ motif containing GTPase activating protein 1.

The proliferation rate of the U87 cells was determined using the MTT assay. As shown in Fig. 2B, the cells transfected with the miR-124a mimic proliferated at a significantly lower rate compared with the blank and NC mimic-transfected cells. In addition, the cell invasion assay revealed that the number of invaded cells was significantly decreased in the miR-124a mimic group compared with the number in the NC mimic group (Fig. 2C).

Effect of IQGAPI knockdown on cell proliferation and invasion. U87 cells were transfected with siRNA to knock down IQGAP1 expression. As shown in Fig. 3A and B, IQGAP1 expression was successfully reduced by siRNAs as determined using real-time PCR and western blot analyses.

Next, cells transfected with siRNA\#1 were subjected to the MTT and cell invasion assays. As shown in Fig. 3C and D, U87 cells with reduced IQGAP1 showed decreased cell proliferation and invasion ability in comparison to the control cells.

IQGAP1 is a direct target of miR-124a in the U87 cells. In order to further validate the relationship between miR-124a and IQGAP1, we carried out the IQGAP1 3'UTR reporter assay in U87 cells. The results revealed that luciferase activity was significantly decreased in the U87 cells transfected with the miR-124a mimic and wild-type IQGAP1-3'UTR, but no reduction was observed in the mutant IQGAP1-3'UTR (Fig. 4). The results demonstrated that IQGAP1 is a direct target of miR-124a.

Effect of IQGAPI knockdown on the expression of $\beta$-catenin and cyclin $D 1$. We tested whether IQGAP1 knockdown could

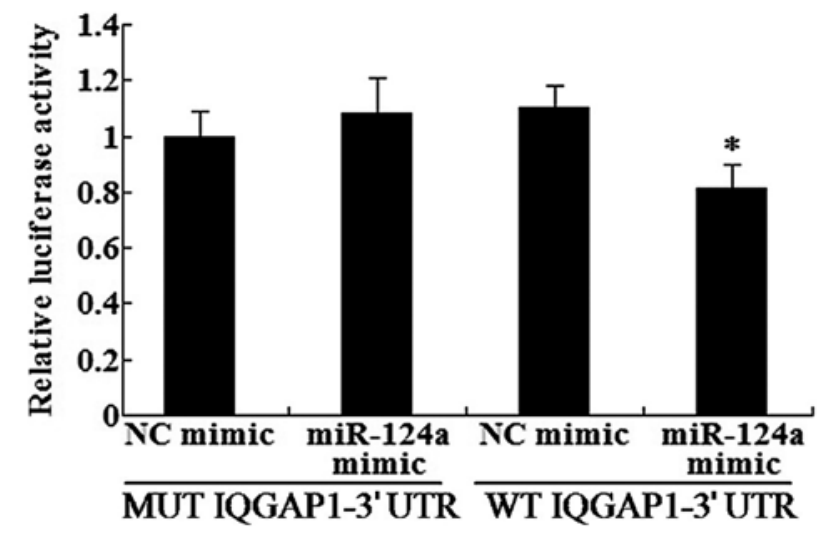

Figure 4. Luciferase assay in the U87 cells cotransfected with wild-type or mutant IQGAP1-3'UTR and NC mimic or miR-124a mimic. ${ }^{*} \mathrm{P}<0.05$ vs. WT IQGAP1-3'UTR+NC mimic group. IQGAP1, IQ motif containing GTPase activating protein 1 .

affect the expression of $\beta$-catenin and cyclin D1. As shown in Fig. 5A, western blot analysis revealed that IQGAP1 knockdown resulted in decreased expression of $\beta$-catenin and cyclin D1.

In addition, immunofluorescence staining showed that in the IQGAP1 siRNA group, $\beta$-catenin was localized in the cytoplasm, and the staining of $\beta$-catenin was weaker in the IQGAP1 knockdown cells compared with the staining in the control cells (Fig. 5C).

miR-124a restoration downregulates the expression of $I Q G A P 1, \beta$-catenin and cyclin DI. We examined the effects of 

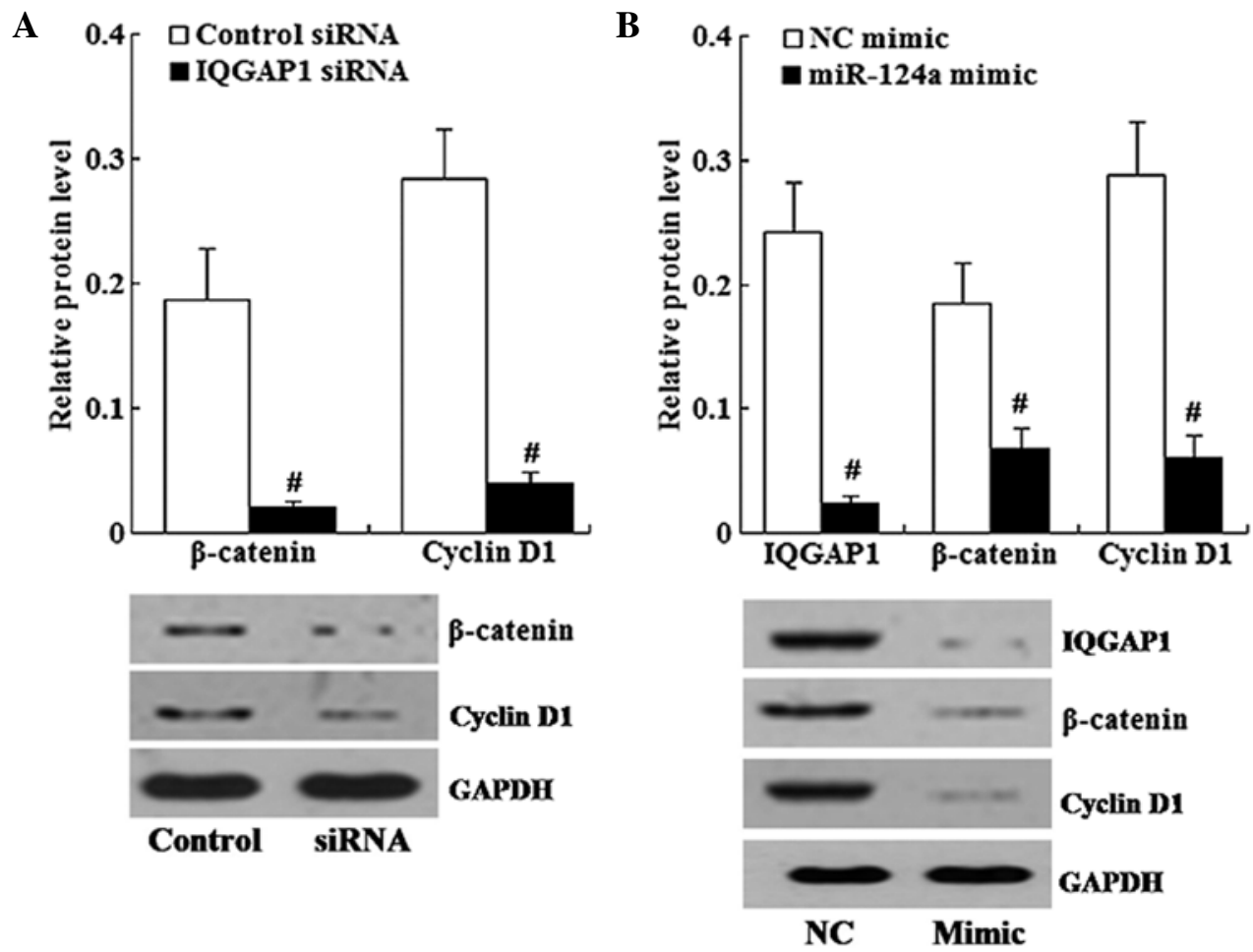

C
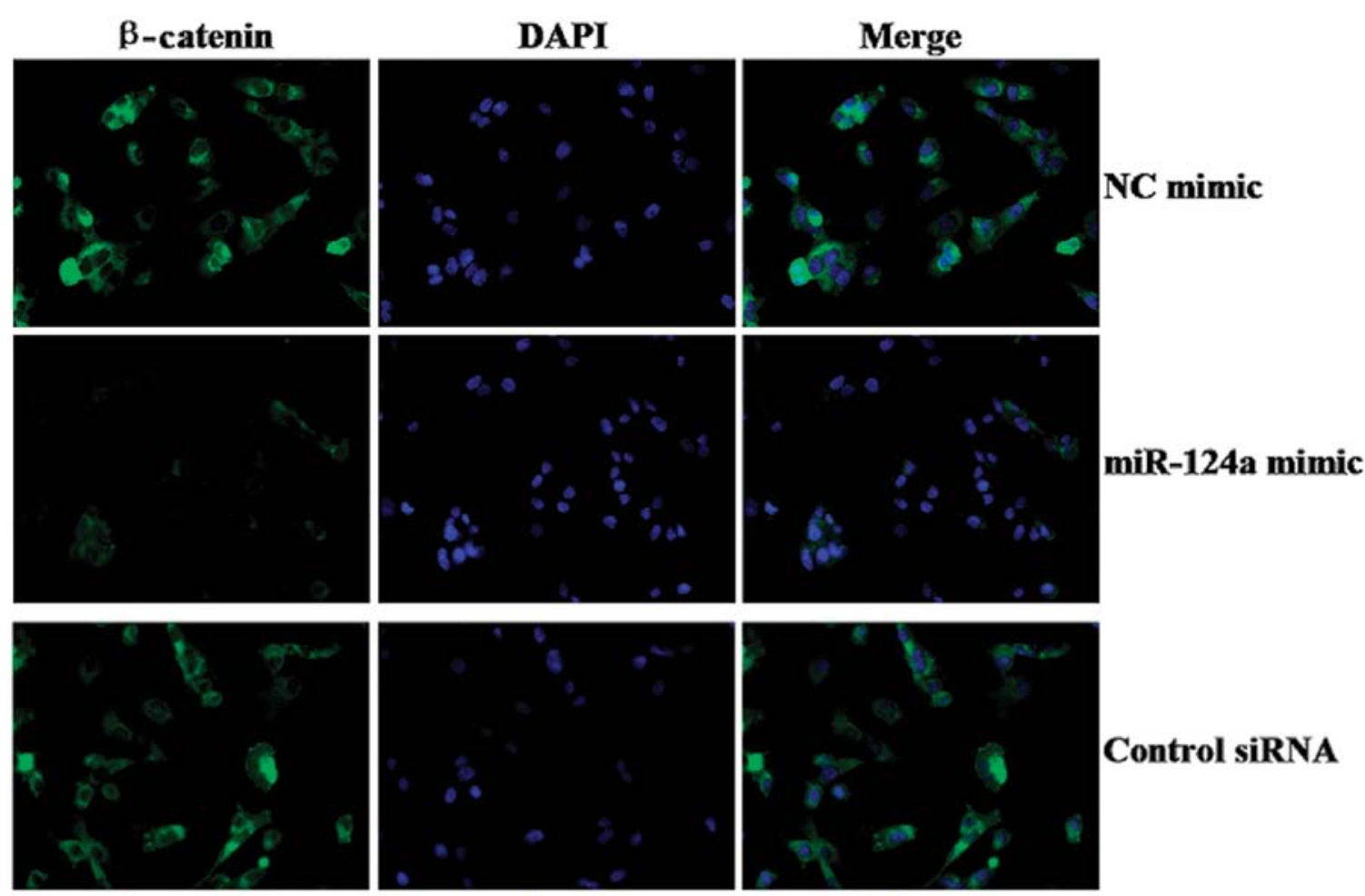

\section{Control siRNA}
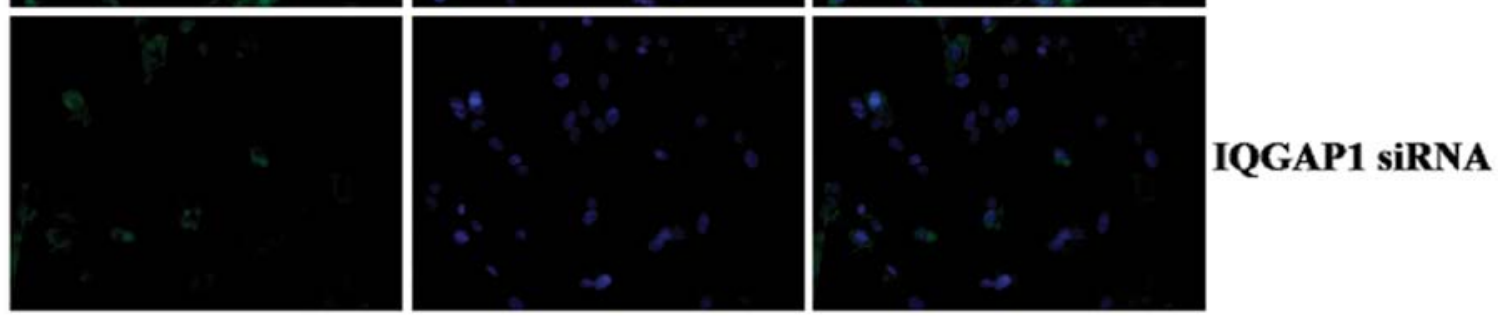

Figure 5. Effect of IQGAP1 knockdown and miR-124a restoration on the expression of downstream proteins. (A) Effect of IQGAP1 knockdown on the expression of $\beta$-catenin and cyclin D1 as determined by western blot analysis. (B) Effect of miR-124a restoration on the expression of IQGAP1, $\beta$-catenin and cyclin D1 as determined by western blot analysis. (C) Immunofluorescence staining of $\beta$-catenin (green) in U87 cells transfected with IQGAP1 siRNA\#1 or the miR-124a mimic. Nuclear DNA was stained with DAPI (blue). Magnification, $\mathrm{x} 400 .{ }^{\#} \mathrm{P}<0.01$ vs. the control group. IQGAP1, IQ motif containing GTPase activating protein 1 ; miR-124a, microRNA-124a. 
miR-124a restoration on the expression of IQGAP1, $\beta$-catenin and cyclin D1. Western blot analysis demonstrated that miR-124a restoration led to decreased expression of IQGAP1, $\beta$-catenin and cyclin D1 (Fig. 5B).

As shown in Fig. 5C, immunofluorescence staining of $\beta$-catenin revealed that compared with the miR-124a NC-transfected cells, $\beta$-catenin was localized in the cytoplasm and the staining of $\beta$-catenin was weaker in the miR-124a mimic-transfected cells.

\section{Discussion}

MicroRNAs (miRNAs) are a class of $\sim 22$ nt long non-coding RNAs which regulate target mRNAs by interacting with the 3'UTR (8). They play important roles in numerous biological processes, such as proliferation, differentiation, development, immunology and cell death (9-14). An increasing number of studies have revealed that aberrant miRNA expression is related to cancer biology, acting as either tumor suppressors or oncogenes $(4,5,15)$.

microRNA-124 (miR-124) has been classified as a tumor suppressor in several types of human cancers (16-18). Previous research has shown that miR-124 is abundantly expressed in normal brain tissue (6), is involved in embryonic neuronal differentiation (19), and is an important regulator of adult neurogenesis in the subventricular zone stem cell niche (20). The present study demonstrated that miR-124a expression is downregulated in human glioma tissues and its expression level is negatively correlated with the pathological grade of glioma. Furthermore, the in vitro study revealed that miR-124a restoration inhibited glioma cell proliferation and invasion. This indicates that miR-124a acts as a tumor suppressor in gliomas.

IQ motif containing GTPase activating protein 1 (IQGAP1) is a member of the IQGAP family. It regulates actin dynamics and cell motility $(21,22)$ by interacting with cytoskeleton components, cell adhesion molecules and several signaling molecules (23). IQGAP1 is important for normal cellular function and homeostasis. Amplification and overexpression of IQGAP1 were reported to be associated with certain malignancies (24-28). IQGAP1 localizes to sites of cell-cell contact (29) and regulates cell-cell adhesion via interacting with E-cadherin and $\beta$-catenin. $\beta$-catenin is the central denominator of the Wnt pathway and plays roles in various types of cancer (30). Cytoplasmic $\beta$-catenin is associated with APC and Axin and forms a destruction complex on which $\beta$-catenin is phosphorylated by the kinases CK $1 \alpha$ and GSK3 $\beta$, leading to $\beta$-catenin degradation by the ubiquitin-proteasome mechanism. Disruption of $\beta$-catenin degradation inhibits $\beta$-catenin phosphorylation and leads to the translocation of $\beta$-catenin into nuclei, where it activates an array of target genes and cell cycle regulators such as c-myc and cyclin D1 $(31,32)$. Given the important role of $\beta$-catenin in cancer, it is of particular interest to identify regulators that may interfere with $\beta$-catenin signaling and thereby lead to cancer development, in particular those miRNAs that can simultaneous interact with multiple regulators of the $\beta$-catenin pathway. In the present study, examination of clinical samples of gliomas showed that IQGAP1 and $\beta$-catenin were upregulated in the glioma tissues, while phospho- $\beta$-catenin was downregulated in the glioma tissues. Furthermore, the in vitro study revealed that IQGAP1 knockdown led to decreased cell proliferation and invasion. Next, molecular mechanisms underlying glioma cell proliferation and invasion were studied. RNA interference assay showed that IQGAP1 regulated the expression of $\beta$-catenin in glioma cells. IQGAP1 knockdown resulted in the downregulation of $\beta$-catenin and cyclin D1. Luciferase assay demonstrated that IQGAP1 is a direct target of miR-124a. Overall, the present study demonstrated that miR-124a inhibits cell proliferation and invasion in glioma cells by targeting IQGAP1, consequently suppressing $\beta$-catenin and downstream cyclin D1.

Our study uncovered a novel molecular mechanism underlying glioma cell proliferation and invasion and may provide a useful molecular therapy for gliomas.

\section{References}

1. Dolecek TA, Propp JM, Stroup NE and Kruchko C: CBTRUS statistical report: primary brain and central nervous system tumors diagnosed in the United States in 2005-2009. Neuro Oncol 14 (Suppl 5): vl-v49, 2012.

2. Louis DN, Ohgaki H, Wiestler OD, et al: The 2007 WHO classification of tumours of the central nervous system. Acta Neuropathol 114: 97-109, 2007.

3. Volinia S, Calin GA, Liu CG, et al: A microRNA expression signature of human solid tumors defines cancer gene targets. Proc Natl Acad Sci USA 103: 2257-2261, 2006.

4. Esquela-Kerscher A and Slack FJ: Oncomirs - microRNAs with a role in cancer. Nat Rev Cancer 6: 259-269, 2006.

5. Hammond SM: MicroRNAs as oncogenes. Curr Opin Genet Dev 16: 4-9, 2006.

6. Lagos-Quintana M, Rauhut R, Yalcin A, Meyer J, Lendeckel W and Tuschl T: Identification of tissue-specific microRNAs from mouse. Curr Biol 12: 735-739, 2002.

7. An L, Liu Y, Wu A and Guan Y: microRNA-124 inhibits migration and invasion by down-regulating ROCK1 in glioma. PLoS One 8: e69478, 2013.

8. Bartel DP: MicroRNAs: genomics, biogenesis, mechanism, and function. Cell 116: 281-297, 2004.

9. Li Q, Bian S, Hong J, et al: Timing specific requirement of microRNA function is essential for embryonic and postnatal hippocampal development. PLoS One 6: e26000, 2011.

10. Chen CZ, Li L, Lodish HF and Bartel DP: MicroRNAs modulate hematopoietic lineage differentiation. Science 303: 83-86, 2004.

11. Brennecke J, Hipfner DR, Stark A, Russell RB and Cohen SM: bantam encodes a developmentally regulated microRNA that controls cell proliferation and regulates the proapoptotic gene hid in Drosophila. Cell 113: 25-36, 2003.

12. Chen $\mathrm{CH}$, Guo M and Hay BA: Identifying microRNA regulators of cell death in Drosophila. Methods Mol Biol 342: 229-240, 2006.

13. Fu LL, Wen X, Bao JK and Liu B: MicroRNA-modulated autophagic signaling networks in cancer. Int J Biochem Cell Biol 44: 733-736, 2012.

14. Ambros V: The functions of animal microRNAs. Nature 431: 350-355, 2004.

15. Medina PP and Slack FJ: microRNAs and cancer: an overview. Cell Cycle 7: 2485-2492, 2008.

16. Xia J, Wu Z, Yu C, et al: miR-124 inhibits cell proliferation in gastric cancer through down-regulation of SPHK1. J Pathol 227: 470-480, 2012.

17. Lang Q and Ling C: MiR-124 suppresses cell proliferation in hepatocellular carcinoma by targeting PIK3CA. Biochem Biophys Res Commun 426: 247-252, 2012.

18. Lv XB, Jiao Y, Qing Y, et al: miR-124 suppresses multiple steps of breast cancer metastasis by targeting a cohort of pro-metastatic genes in vitro. Chin J Cancer 30: 821-830, 2011.

19. Cao X, Pfaff SL and Gage FH: A functional study of miR-124 in the developing neural tube. Genes Dev 21: 531-536, 2007.

20. Cheng LC, Pastrana E, Tavazoie M and Doetsch F: miR-124 regulates adult neurogenesis in the subventricular zone stem cell niche. Nat Neurosci 12: 399-408, 2009.

21. Watanabe T, Wang S, Noritake J, et al: Interaction with IQGAP1 links APC to Rac1, Cdc42, and actin filaments during cell polarization and migration. Dev Cell 7: 871-883, 2004. 
22. Mataraza JM, Briggs MW, Li Z, Entwistle A, Ridley AJ and Sacks DB: IQGAP1 promotes cell motility and invasion. J Biol Chem 278: 41237-41245, 2003.

23. Nieto MA: The snail superfamily of zinc-finger transcription factors. Nat Rev Mol Cell Biol 3: 155-166, 2002.

24. Nabeshima K, Shimao Y, Inoue T and Koono M: Immunohistochemical analysis of IQGAP1 expression in human colorectal carcinomas: its overexpression in carcinomas and association with invasion fronts. Cancer Lett 176: 101-109, 2002.

25. Dong P, Nabeshima K, Nishimura N, Kawakami T, Hachisuga T, Kawarabayashi $\mathrm{T}$ and Iwasaki $\mathrm{H}$ : Overexpression and diffuse expression pattern of IQGAP1 at invasion fronts are independent prognostic parameters in ovarian carcinomas. Cancer Lett 243 : 120-127, 2006

26. Balenci L, Clarke ID, Dirks PB, et al: IQGAP1 protein specifies amplifying cancer cells in glioblastoma multiforme. Cancer Res 66: 9074-9082, 2006.
27. Johnson M, Sharma M and Henderson BR: IQGAP1 regulation and roles in cancer. Cell Signal 21: 1471-1478, 2009.

28. Nakamura H, Fujita K, Nakagawa H, Kishi F, Takeuchi A, Aute I and Kato H: Expression pattern of the scaffold protein IQGAP1 in lung cancer. Oncol Rep 13: 427-431, 2005.

29. Kuroda S, Fukata M, Nakagawa M, et al: Role of IQGAP1, a target of the small GTPases Cdc42 and Rac1, in regulation of E-cadherin-mediated cell-cell adhesion. Science 281: 832-835, 1998.

30. Polakis P: Wnt signaling and cancer. Genes Dev 14: 1837-1851, 2000.

31. Breuhahn K, Longerich T and Schirmacher P: Dysregulation of growth factor signaling in human hepatocellular carcinoma. Oncogene 25: 3787-3800, 2006.

32. Lee HC, Kim M and Wands JR: Wnt/Frizzled signaling in hepatocellular carcinoma. Front Biosci 11: 1901-1915, 2006. 